\title{
Why does uncertainty come in quantum mechanics?
}

\author{
Muhammad Yasin \\ Savar, Dhaka, Bangladesh \\ E-mail:md.yasin@ymcontents.com
}

1 Abstract: In 1927 Heisenberg has invented the uncertainty principle. The principle of uncertainty is, "It is impossible to determine the position and momentum of a particle at the same time."The more accurately the momentum is measured, the more uncertain the position will be. Just knowing the position would make the momentum uncertain. Einstein was adamant against this principle until his death. He thought that particles have some secret rules. Einstein thought, "The uncertainty principle is incomplete. There is a mistake somewhere that has resulted in uncertainty. Many did not accept Einstein then. But I'm sure Einstein was right then, there are secret rules for particles. Heisenberg's uncertainty principle is also $100 \%$ correct . I recently published a research paper named "Quantum Certainty Mechanics" ${ }^{11]}$, which shows the principle of measuring the momentum and position of particles by the quantum certainty principle. Why uncertainty comes from certainty is the main topic of this research paper. When the value of the energy absorbed by the electron in the laboratory is calculated, the uncertainty is removed. The details are discussed below.

2 Keywords: quantum mechanics; uncertainty principle; certainty mechanics ; quantum measurement

3 Introduction: Basically I have tried to show in two ways, why uncertainty comes. When the particle moves with the momentum $p$, the wave involved with the particle is revealed. Due to the p-momentum of the particle, the distance between the particle and the wave associated with the particle is $\theta>0$ and then the detection of the particle inside the wave leads to uncertainty. If the distance between the particle and the involved wave due to the p momentum of the particle is $\theta=0$, then there would be no uncertainty. But the reality is that as the particle moves with momentum $\mathrm{p}$, there is always $\theta>0$ between the particle and the wave associated with the particle, so there is uncertainty.

It is possible to analyze the matter in another way. When we go to observe a particle in the laboratory, the position of the particle changes due to the effect of the energy of the photon or the energy of another instrument on the electron and gains kinetic energy. Calculating how much energy has been applied while observing the particle in the laboratory will reduce the uncertainty. To avoid uncertainty when measuring the position and momentum of a particle, we 
need to measure how much energy has been absorbed from photon by the electron to make the total energy $\mathrm{E}$. If the total energy $\mathrm{E}$ of a photon can be measured with certainty then the position and momentum of the particle can be measured with certainty. This article will show that the uncertainty principle can be proved from the principle of certainty by diffraction test and the uncertainty principle comes when the distance between the particle and the wave involved is 6> 0 due to the $\mathrm{p}$ momentum of the particle.

4 Method: The recently published article entitled "Quantum Certainty Mechanics" shows that it is possible to prove the principle of certainty $(\mathrm{xp}=\mathrm{hft})$ separately from the theory of relativity, quantum mechanics, Newton's mechanics (maintaining the principle of relativity) and Heisenberg's law of uncertainty. Here $\mathrm{x}$ is the position, $\mathrm{p}$ is the momentum, $\mathrm{f}$ is the frequency of the waves involved due to the $\mathrm{p}$ momentum of the particle and $\mathrm{t}$ is the time. Basically this equation explains why uncertainty comes. The reasons for the uncertainty have been shown in two ways.

\subsection{Mathematical proof of the principle uncertainty from the principle certainty:}

." Now I will tell you why uncertainty comes. Suppose a snake is running in the river. At that time waves are created in the river. Now if I find the snake inside the wave then the position of the snake will be uncertain. But if we consider finding snakes in the waveless river, there will be no uncertainty. A particle is moving. Waves are created or waves are involved because of the motion of the particles. The waves are like beautiful sine waves. Like a snake, we will look for the particle inside the wave, that is, inside the sine function.

The wave is, $\sin (\omega t)$

Now the principle of certainty is,

$$
\begin{aligned}
\mathrm{xp} & =\mathrm{hft} \\
& \Rightarrow \mathrm{xp}=\frac{\mathrm{h}}{2 \pi} \omega \mathrm{t} \\
& \Rightarrow \mathrm{xp}=\hbar \omega \mathrm{t}
\end{aligned}
$$

therefore,

$$
\omega t=\frac{x p}{\hbar}
$$

From equations (1) and (2) we get;

$\sin \left(\frac{\mathrm{xp}}{\hbar}\right)$

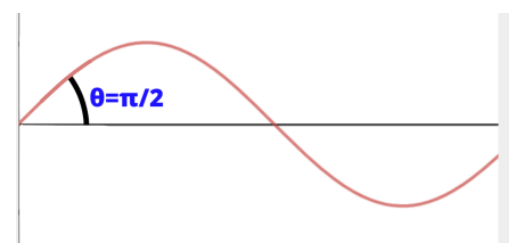

Figure 1: An electron at the main point and the wave created due to the momentum of the electron are depicted.

The value of the wave is, $-1 \leq \sin (\omega t) \leq 1$ 
That is,

$$
\begin{aligned}
\theta & \geq 0 \\
& \Rightarrow \frac{x p}{\hbar} \geq 0
\end{aligned}
$$

So hold the distance $\theta=\omega t \geq \frac{\pi}{2}$ between the electron and the wave at the original point and get from equation (2),

$$
\begin{aligned}
& \frac{x p}{\hbar} \geq \frac{\pi}{2} \\
& \Rightarrow \mathrm{xp} \geq \frac{\pi}{2} \times \frac{\mathrm{h}}{2 \pi} \\
& \Rightarrow \mathrm{xp} \geq \frac{\mathrm{h}}{4}
\end{aligned}
$$

Equation 4 indicates uncertainty. I said a while ago that the principle of certainty is correct. Again I am saying, if the certainty is correct, then the principle of uncertainty is also correct. In fact, the reason for the uncertainty is that I found the electron in $\sin (\theta)$ at $\theta \geq \pi / 2$. That is, when I look for electrons inside the wave, uncertainty will come. If we continue to reduce the value of $\theta$, then the value of uncertainty will continue to decrease. In this way uncertainty can be removed. If the value of $\theta$ reduces to 0 , that is, if the distance between the electron and the wave created by the electron is $\theta=0$ then there is nothing to say about uncertainty. What is uncertainty? Is it very important to stay?

Can't it be omitted in any way? What is the role of the certainty from which the uncertainty came? Will we not get rid of uncertainty in any way?

For this answer we assume Dolphins are running in the sea. For convenience, there is no wave in the sea.

Now when dolphins run, they create waves. The size of each wave is equal to 10 to 20 floors (imagine, there is nothing wrong with imagining!). Now the dolphin is running through waves equal to 10 to 20 floors. I don't understand where the dolphins are. Now you have to look at the sea waves to identify the dolphins. Then we can say that the bigger the wave, the more likely it is that there will be dolphins. When we can't identify the dolphin, instead we go to identify the dolphin by the waves, then the dolphins are everywhere in the waves of the ocean. Just then the uncertainty will come. Similarly, when we cannot identify the electron, when we go to determine the position of the electron through the created (involved) wave due to the movement of the electron, then uncertainty will come. Whether it's finding the dolphin in the waves of the ocean or finding the electron in the waves created with it. The dolphin or the electron is in a certain position but the waves created with it can be in any position. Just then uncertainty shifts from certainty. And if we do not want to bring uncertainty, then the distance between the electron and the created (involved) wave with the electron must be calculated as $\theta=0$. Then there will be no uncertainty 
I have done a little trick in implementing the principle of uncertainty from the principle of certainty.

$$
\sin \left(\frac{x p}{\hbar}\right)
$$

Here we have calculated holding. $\frac{\mathrm{xp}}{\hbar} \geq \frac{\pi}{2}$

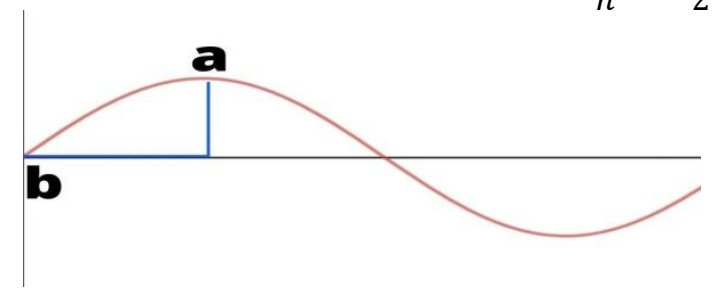

We have calculated from the highest peak in the figure i.e. $\theta=\frac{\pi}{2}$ but we should have calculated from $\theta=0^{\circ}$ first.

Let's calculate the beginning with $\theta=0$.

I have said before that if you reduce $\theta$, the value of uncertainty will continue to decrease. So what will happen now?

Suppose the distance between an electron and a wave created with an electron is $\theta=0^{\circ}$.

$$
\begin{aligned}
& \frac{\Delta x \Delta p}{\hbar} \geq 0 \\
& \Rightarrow \Delta x \Delta p \geq 0
\end{aligned}
$$

There is no further uncertainty in Equation 5.In fact, uncertainty comes when we calculate the value $\theta=\pi / 2$, but if you calculate the value $\theta=0$ then there is no uncertainty. In the universe, when an object moves with p-momentum, its matter-wave properties are revealed. There is always $\theta=\pi / 2$ between these waves and particles which leads to uncertainty.

If $\theta=0{ }^{\circ}$ existed between the particle and the wave associated with the particle, then the uncertainty principle would not work. 


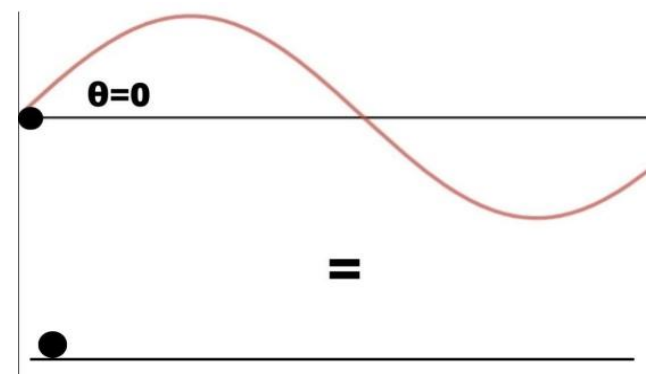

\subsection{Mathematical proof of the principle uncertainty from the principle certainty} (fundamental equation)

Let's find out the general equation of the relationship between the principle of certainty and the principle of uncertainty.

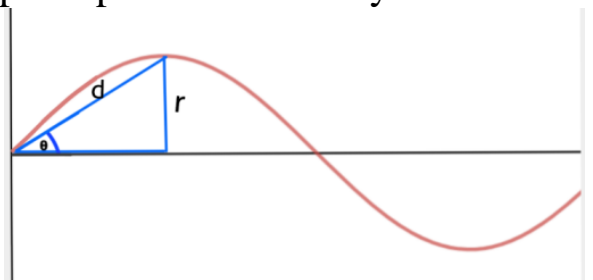

Notice the figure above which is the graph for $\sin (\theta)$.Here

$$
\begin{aligned}
& \sin (\theta)=\frac{r}{d} . \\
& \Rightarrow \sin (\omega t)=\frac{r}{d}
\end{aligned}
$$

Now we know, $\omega t=\frac{x p}{\hbar}$. So we get from equation 48

$$
\begin{aligned}
\sin \left(\frac{x p}{\hbar}\right) & =\frac{r}{d} \\
\Rightarrow \frac{x p}{\hbar} & =\sin ^{-1}\left(\frac{r}{d}\right)
\end{aligned}
$$

Now if we assure the momentum $p$ then the uncertainty of position from the equation 7 will be ,

$$
\Delta \mathrm{x}=\frac{\hbar}{\Delta \mathrm{p}} \sin ^{-1}\left(\frac{\mathrm{r}}{\mathrm{d}}\right)
$$

The reason for the uncertainty is that if the distance between the electrons and the waves associated with the electrons (created waves) is calculated more, the uncertainty comes. Whether the uncertainty will be more or less will depend on the following equation.

$$
\Delta \mathrm{x} \propto \sin ^{-1}\left(\frac{\mathrm{r}}{\mathrm{d}}\right)
$$

The mystery of whether uncertainty will increase or decrease is hidden in this equation 9. 
I already told why the uncertainty come.

Let's take a look at the relationship between the principle of uncertainty and the principle of certainty according to Broglie's particle-wave duality.

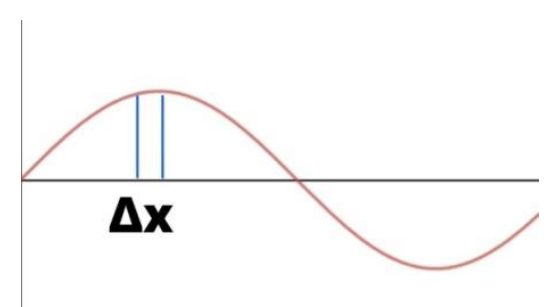

If we are sure of $\Delta x$ then we do not know what will be $\lambda$. Then according to the $\lambda=\frac{h}{p}$ formula $\Delta x$ is sure but the momentum $\Delta \mathrm{p}$ is uncertain.

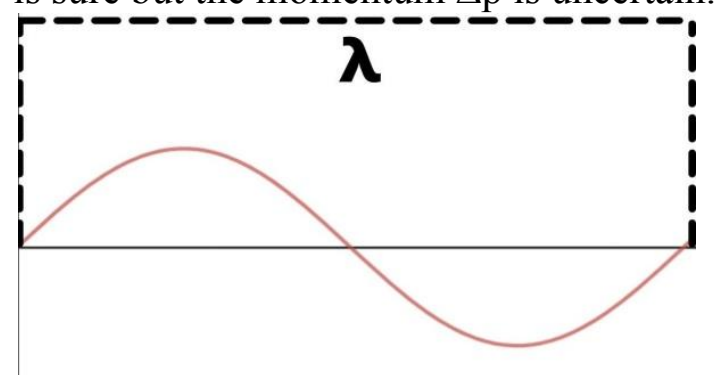

Now $\lambda$ confirmation means that according to the formula $p=\frac{h}{\lambda}$ the momentum $\mathrm{p}$ is certain but $\Delta \mathrm{x}$ is then uncertain. Then what is the matter!

We have seen from the principle of certainty that if it is $\theta \geq \frac{\pi}{2}$ then uncertainty comes. Which means I've been trying to find the electrons inside the waves. $\lambda$ Confirmation in the Broglie's formula and $\theta \geq \frac{\pi}{2}$ in the principle of certainty is the same thing. Then this is the background behind the coming of uncertainty.

\subsection{The proof of uncertainty from the principle of certainty through deflection:}

With the help of the electron deflection system it is possible to prove the principle of uncertainty.

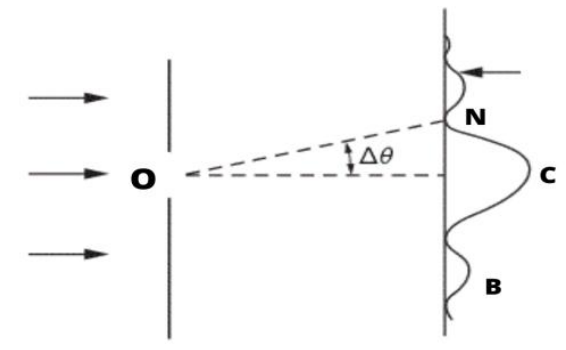

The figure above, where a beam of an electron of $\mathrm{v}_{0}$ velocity is falling on a screen $\mathrm{A}$ with a hole of $\Delta \mathrm{x}$ thickness from the left side. In this case the process of deflection will occur due to the wave nature associated with the electron and hence the deflection pattern will be created in 
screen B placed parallel to it. Suppose the value of the perpendicular (x) component $\mathrm{v}_{\mathrm{x}}$ of the velocity of the electron emitted from the hole at point $\mathrm{N}$ on the screen $\mathrm{B}$ is $\mathrm{V}_{\mathrm{xN}}$, which in this case indicates the uncertainty of velocity, i.e. $V_{x N}=\Delta v_{x}$. Now you can write from the figure.

$$
\begin{aligned}
\tan \left(\frac{C N}{O C}\right) & \\
\Rightarrow \theta & =\frac{\left(v_{x N}\right) t}{\left(v_{o}\right) t} \\
\Rightarrow \theta & =\frac{\left(v_{x N}\right)}{\left(v_{o}\right)} \\
\therefore \theta & =\frac{\Delta v}{v_{o}}
\end{aligned}
$$

(Because, if $\theta$ is small then $\tan (\theta) \cong \theta$ )

Now we get multiplied by mc on both sides,

$$
\begin{aligned}
\theta= & \frac{\Delta \mathrm{v} \times \mathrm{mc}}{\mathrm{v}_{\mathrm{o}} \times \mathrm{mc}} \\
& \Rightarrow \theta=\frac{\Delta \mathrm{E}}{\mathrm{E}}
\end{aligned}
$$

Now according to the principle of certainty, $\Delta \mathrm{x} \Delta \mathrm{p}=\mathrm{hft}$

$\therefore \mathrm{xp}=\mathrm{hft}$

$$
\begin{aligned}
& \Rightarrow \mathrm{xp}=\frac{\mathrm{h}}{2 \pi} 2 \pi \mathrm{ft} \\
& \Rightarrow \mathrm{xp}=\hbar \theta \\
& \Rightarrow \theta=\frac{\mathrm{xp}}{\hbar}
\end{aligned}
$$$$
[2 \pi \mathrm{ft}=\theta]
$$

Now we get from Equation 11 and Equation 12,

$$
\begin{aligned}
& \frac{\Delta \mathrm{E}}{\mathrm{E}}=\frac{\mathrm{xp}}{\hbar} \\
& \Delta \mathrm{x} \Delta \mathrm{p}=\hbar \frac{\Delta \mathrm{E}}{\mathrm{E}}
\end{aligned}
$$

In equation no 13 , the nature of the uncertainty will depend on $\Delta \mathrm{E} / \mathrm{E} . \mathrm{E}$ is the total energy of the electron, the static energy and the total energy due to the impact of the photon. $\Delta \mathrm{E}$ is the erroneous value of measuring $\mathrm{E}$. $\Delta \mathrm{E}=\mathrm{E}$ when the energy of the electron is measured in a $0 \%$ certain way i.e. $100 \%$ uncertain energy $\Delta \mathrm{E}$ is measured. Then the momentum and position are $100 \%$ uncertain. Again, if we measure the energy of the electron accurately, that is, if we measure it $100 \%$ surely, $0 \%$ will be uncertain, that is, $\Delta \mathrm{E}=0$. Then the uncertainty of momentum and position will be $0 \%$ i.e. $\Delta \mathrm{x} \Delta \mathrm{p}=0$.

Which means that if the value of energy uncertainty $\Delta \mathrm{E}$ increases to $\Delta \mathrm{E}=\mathrm{E}$ then $\Delta \mathrm{x} \Delta \mathrm{p}$ will be completely uncertain. If we can accurately measure the energy of an electron, then the uncertainty of the value of $\Delta x \Delta p$ will continue to decrease. The uncertainty of the energy of the 
electron at point $\mathrm{N}$ in the figure above is $\Delta \mathrm{E}$. So the uncertainty of momentum and position exists .When we do experiment to see electrons, the energy of electrons increases under the influence of laboratory instruments. But we do not calculate the value of $\Delta \mathrm{E}$. Whereby the value of $\Delta \mathrm{E}$ is equal to the completely uncertain total energy $E$.Then the uncertainty of $\Delta x \Delta p$ arises.

5 Decision: Uncertainty arises when the momentum and position of a particle are measured simultaneously. The main reason for the uncertainty is our observation effect. Uncertainty arises when the distance between the particle and waves created due to the $p$ momentum of the particle is $\theta>0$.If the distance between the particle and the wave caused by the $p$-momentum of the particle were $\theta=0$, there would be no uncertainty. According to the experimental results, the total energy $(\Delta \mathrm{E})$ of the particle at any point is uncertain when observing the particle in the laboratory, As a result $\Delta x \Delta p$ at that point becomes uncertain. If we determine the total energy $E$ of the particle in the laboratory, then there will be no uncertainty. Einstein has been against the principle of uncertainty all his life since 1927, according to him there is a secret rule. This article reveals that secret rule.

6 Conclusion: The uncertainty principle is completely correct. The main purpose of this article is to show how the principle of uncertainty comes from the principle of certainty. Einstein's claim was correct that day, there is a reason behind the uncertainty principle. How or why the uncertainty principle comes can be known for sure from the principle of certainty .

\section{Reference}

[1] Yasin ,M . Quantum Certainty Mechanics (2021) osf.oi

[2] Ozawa, M: Heisenberg's Original Derivation of the Uncertainty Principle and its Universally Valid Reformulations (2015).arXiv:1507.02010

[3] Busch , P: Heisenberg's uncertainty principle (2007). arXiv:quant-ph/0609185v3

[4] W. Heisenberg, Remarks on the origin of the relations of uncertainty, in: W. Price, S. Chissick(Eds.), The Uncertainty Principle and Foundations of Quantum Mechanics. A Fifty Years'Survey, J. Wiley \& Sons, London, 1977, pp. 3-6.

[5] W. Heisenberg, Uber den anschaulichen Inhalt der quantentheoretischen $\mathrm{Ki}$ " nematik und Mechanik, Z. Phys. 43 (1927) 172-198.

[6] P. Busch, C.R. Shilladay, Complementarity and uncertainty in Mach-Zehnder interferometry and beyond, Phys. Rep. 435 (2006) 1-31.

[7] Planck M 1900 Zur Theorie des Gesetzes der Energieverteilung im NormalspectrumVerhandlungen der Deutschen Physikalischen Gesellschaft2, 237-45,English translation by D. ter Haar 1967The Old Quantum Theory(PergamonPress]:

[8] https://en.wikipedia.org/wiki/Uncertainty_principle.

[9] Heisenberg, W. The physical content of quantum kinematics and mechanics. In Wheeler, J. A. \& Zurek, W. H. (eds.) Quantum Theory and Measurement, 62-84 (Princeton UP, Princeton, NJ.

[10]Ozawa, M. Noise and disturbance in quantum measurements and operations. Proc. SPIE 6244, 62440Q (2006). 
[11] Lund, A. P. \& Wiseman, H. M. Measuring measurementdisturbance relationships with weak values. New J. Phys. 12, 093011 (2010).

[12] Ozawa, M. Uncertainty relations for noise and disturbance in generalized quantum measurements. Ann. Phys. (N.Y.) 311, 350416 (2004).

[13] Ozawa, M: Heisenberg's Original Derivation of the Uncertainty Principle and its Universally Valid Reformulations (2015).arXiv:1507.02010

[14] https://en.wikipedia.org/wiki/Uncertainty_principle.

[15] Heisenberg, W. The physical content of quantum kinematics and mechanics. In Wheeler, J. A. \& Zurek, W. H. (eds.) Quantum Theory and Measurement, 62-84 (Princeton UP, Princeton, NJ.

[16]Ozawa, M. Noise and disturbance in quantum measurements and operations. Proc. SPIE 6244, 62440Q (2006).

[17] Lund, A. P. \& Wiseman, H. M. Measuring measurementdisturbance relationships with weak values. New J. Phys. 12, 093011 (2010).

[18] Ozawa, M. Uncertainty relations for noise and disturbance in generalized quantum measurements. Ann. Phys. (N.Y.) 311, 350416 (2004). [119] A. Einstein, B. Podolsky, N. Rosen, Can quantum-mechanical description of physical reality be considered complete?, Phys. Rev. 47 (1935) 777-780 